\title{
The 1st International Workshop on Analytics Services on the Cloud (ASC 2012)
}

This proceeding contains all the papers that were selected for presentation at the First International Workshop on Analytics Services on the Cloud (ASC 2012), which was held in Shanghai, China, November 12, 2012.

Every day, quintillions of bytes of data are created in different domains, including Internet, healthcare, retail and so on. The term "big data" is used to describe such data, which has three major characteristics: large volume, high speed of data flow and heterogeneous data types. Studies have shown that leveraging knowledge from big data via analytics will generate tremendous business impact. In the foreseeable future, the use of big data will become a key basis of competition for enterprises.

With the needs of analytics growing at ever increasing speeds, it becomes clear that traditional data processing techniques are in no way able to meet the demands. The use of big data has raised tremendous challenges on analysis techniques, e.g., data integration and linkage, data storage, pattern mining, IT infrastructure to support massive and scalable data processing, and visualization of the analysis results. Therefore, Cloud Computing becomes a perfect candidate for delivering big data analytics. However, the emerging Cloud Computing technology adds another interesting dimension in this complex area since Cloud technology enables a new service model when delivering analytics to end users.

To provide a balanced coverage and an equal emphasis across all aspects of analytics services on the cloud, ASC 2012's topic were divided in to three major areas: performance, service infrastructure and applications. We solicited 15 high quality submissions. Only 7 papers were accepted for the program. The paper "Kachako: a Hybrid-Cloud Unstructured Information Platform for Full Automation of Service Composition, Scalable Deployment and Evaluation", written by Yoshinobu Kano, was selected for the best paper award sponsored by IBM Research.

This outstanding workshop program was a testament to the efforts of many dedicated individuals who were committed to the success of ASC 2012. This success would not have been possible without the enthusiastic support from the Program Committee, the efficient organizational efforts from the ICSOC 2012 workshop organizers and the significant contributions from the authors who submitted their work to ASC 2012. They all deserve our deep gratitude for the efforts they have made.

Yu Deng, Liangzhao Zeng IBM T.J. Watson Research Center P.O. Box 704

Yorktown Heights, NY 10598 USA dengy@us.ibm.com, lzeng@us.ibm.com 


\section{Organization}

\section{Workshop Officers}

\section{General Chair}

- Yu Deng, IBM Research - Watson Lab, USA

- $\quad$ Liangzhao Zeng, IBM Research - Watson Lab, USA

\section{Presentation Chair}

- Minkyong Kim, IBM Research - Watson Lab, USA

\section{Program Committee}

- Han Chen, IBM Research - Watson Lab, USA

- $\quad$ Shiping Chen, CSIRO ICT Centre, Australia

- Liana Fong, IBM Research - Watson Lab, USA

- Lipyeow Lim, University of Hawaii at Manoa, USA

- Marco Aurelio Stelmar Netto, IBM Research - Brazil Lab, Brazil

- Vugranam Sreedhar, IBM Research - Watson Lab, USA

- Timothy Wood, George Washington University, USA

- Xifeng Yan, University of California at Santa Barbara, USA

- Wlodek Zadrozny, IBM Research - Watson Lab, USA

- $\quad$ Sai Zeng, IBM Research - Watson Lab, USA

- Youhui Zhang, Tsinghua University, China

- $\quad$ Shuigeng Zhou, Fudan University, China 